\title{
Pancreatic ductal cells in development, regeneration, and neoplasia
}

\author{
Maximilian Reichert and Anil K. Rustgi
}

Division of Gastroenterology, Department of Medicine, Abramson Cancer Center, University of Pennsylvania School of Medicine, Philadelphia, Pennsylvania, USA.

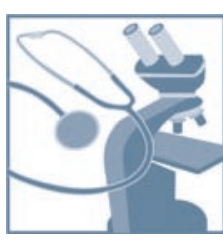

The pancreas is a complex organ comprised of three critical cell lineages: islet (endocrine), acinar, and ductal. This review will focus upon recent insights and advances in the biology of pancreatic ductal cells. In particular, emphasis will be placed upon the regulation of ductal cells by specific transcriptional factors during development as well as the underpinnings of acinar-ductal metaplasia as an important adaptive response during injury and regeneration. We also address the potential contributions of ductal cells to neoplastic transformation, specifically in pancreatic ductal adenocarcinoma.

\section{Introduction}

The pancreas is an organ that has endocrine and exocrine compartments (1). The endocrine compartment consists of $\alpha, \beta, \delta, \varepsilon$, and pancreatic polypeptide cells organized into islets, whereas the exocrine compartment consists of acinar, ductal, and centroacinar cells (Figure 1). Acinar cells synthesize and secrete digestive enzymes, which are concentrated into a bicarbonate rich fluid that traverses through a complex network of ducts (Figure 1). The terminal, or intercalated, ducts are lined by flat, almost squamous-like epithelia. Terminal end duct cells that interface with acini are called centroacinar cells (2). Intercalated ducts merge to form intralobular ducts (lined by cuboidal epithelia), and these in turn merge to form interlobular ducts, which finally merge to form into the main duct (lined by simple columnar epithelia) that traverses the pancreas to the duodenum, delivering fluid laden with digestive enzymes.

This review will focus initially on what is known about the role of specific transcriptional factors that govern ductal cell morphogenesis and biology during development and in adult tissues. Second, we will address acinar-ductal metaplasia (ADM), which is an important component of pancreatitis and neoplastic transformation. Understanding the governance of ADM provides potential insights into ductal cell morphogenesis. Finally, how ductal cells might contribute to neoplastic transformation will be discussed. The field of ductal cell biology is still evolving, and here we place what is known into the context of what still needs to be discovered. For some recent comprehensive reviews on the endocrine and exocrine lineages during development as well as their physiological functions in the adult pancreas, see refs. 3-7.

\section{Regulation of pancreatic development}

The pancreas, along with the liver and biliary tract, arises from a shared multipotent population of cells in the foregut endoderm $(8,9)$. In the human, the pancreas is first discernible as the dorsal bud that emerges from the proximal duodenum at four weeks of gestation. A collection of primitive epithelial tubules within the pancreas is evident at week seven. The epithelial tubules comprise central ducts that have an admixture of loose mesenchyme and peripheral ducts encompassed by a peripancreatic mesenchyme. These tubules undergo branching, and mature acini develop from

Conflict of interest: The authors have declared that no conflict of interest exists. Citation for this article: J Clin Invest. 2011;121(12):4572-4578. doi:10.1172/JCI57131. cell buds that surround their ends. Development of endocrine cells begins in the central duct area and, with increasing developmental age, moves toward the periphery. Endocrine cells are apparent in the developing islets after 10 weeks of gestation. During the third month of gestation, both mature secretory acini and islets of Langerhans can be recognized.

In the mouse, the earliest morphological evidence of the pancreas arises around E8.5 to E9.5. The ventral and dorsal pancreatic buds fuse as a result of gut tube rotation around E13. Up to this point, the pancreas consists of protodifferentiated epithelium (termed pancreatic cords or pancreatic trunk epithelium). During a time termed "secondary transition" (starting around E13.5 to E14.5), a burst of proliferation accompanied by differentiation occurs (4). Histologically distinct acinar and ductal cells do not appear until approximately E15 (10).

Pancreatic development is a tightly regulated process, with the endocrine and exocrine compartments emerging from a common progenitor population. This process involves the interplay of Hedgehog signaling during early pancreatic development (11-13), Notch signaling, and other cues from the mesenchyme (14-17). In addition, genetic studies have identified a number of transcription factors critical for pancreatic development; notably, $\mathrm{Pdx} 1$ is required for the specification of all pancreatic lineages (18-20), and Pdx1, ngn3, NeuroD (also known as BETA2), Hnf6, and Pax4 all contribute to proliferation, differentiation, and endocrine lineage commitment (20-26). Exocrine lineage specification or differentiation is influenced both by the lack of proendocrine transcription factors and by the presence of permissive signals furnished by contiguous pancreatic mesenchyme $(12,15,27)$, including Wnt signaling (28), laminin-1, and soluble follistatin $(29,30)$. Furthermore, the levels of FGF-1, FGF-7, and TGF- $\beta_{1}$, activin and EGFR are important in determining the balance between endocrine and exocrine differentiation (31-34). Notably, these findings are based upon ex vivo organ cultures.

Acinar cell differentiation during development appears to be regulated by the bHLH transcription factor, Ptf1a (also known as p48) (35-37). Although detected early in pancreatic development in multipotent progenitor cells, Ptf1a expression becomes restricted to differentiating and mature acinar cells (18). Knockout of Ptfla in mice leads to an absence of the exocrine pancreas and displacement of islet cells to the spleen, in which the endocrine compartment resides in some lower vertebrates (38). However, 
A

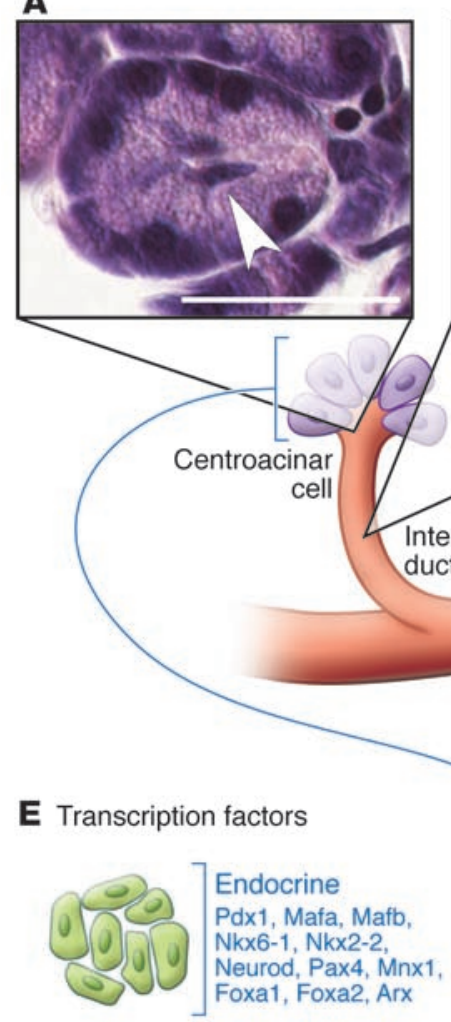

B
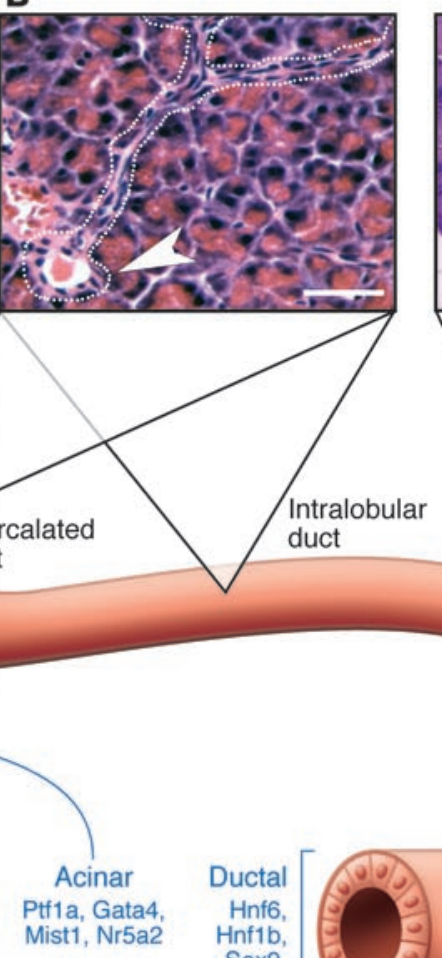

Mist1, Nr5a2
C

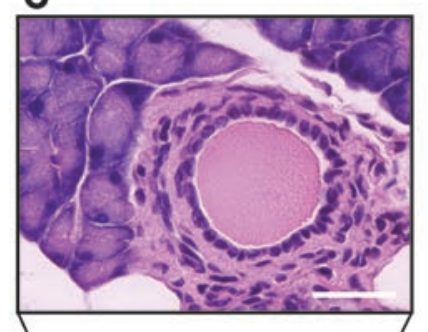

D

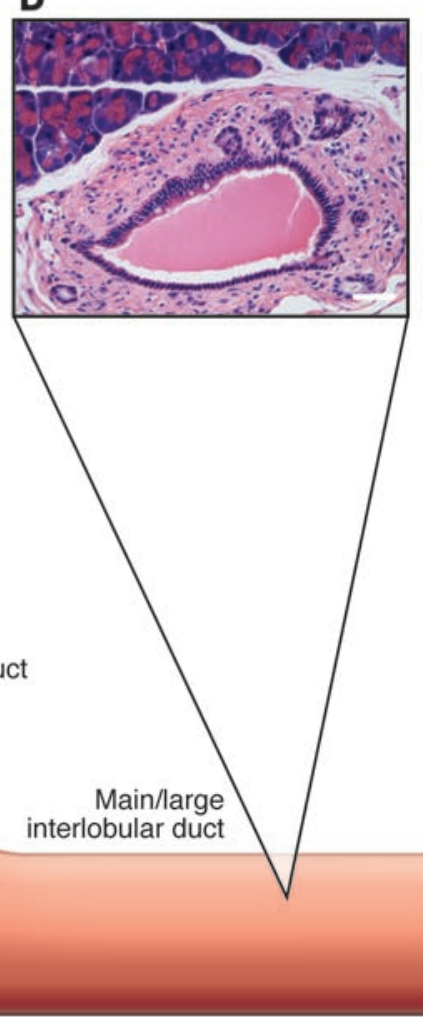

Figure 1

Anatomical organization of the pancreatic ductal tree. (A) Centroacinar cells are terminal end duct cells that interface with acini (white arrowhead; scale bar: $10 \mu \mathrm{m}$ ). (B) Terminal ducts or intercalated ducts (dotted line), respectively, are composed of flat epithelia and merge into intralobular ducts (white arrowhead) that are lined by cuboidal epithelia (scale bar: $10 \mu \mathrm{m}$ ). (C) Intralobular ducts merge to form small interlobular ducts surrounded by mesenchyme (scale bar: $10 \mu \mathrm{m}$ ). (D) Larger interlobular ducts are lined by columnar epithelia (scale bar: $10 \mu \mathrm{m})$. (E) Some key acinar, ductal, and endocrine cell transcription factors are depicted (see refs. 4,6 ).

studies in similar mouse models reveal the persistence of early endocrine cells closely associated with the pancreatic ductal remnant $(18,35,39)$. Mist 1 is another bHLH transcription factor that becomes important at the approximate time of secondary transition, and, as elaborated upon below, mice lacking Mist 1 exhibit defective acinar cellular organization (40).

\section{Regulation of the ductal cell lineage during development}

Ductal cells express markers such as cytokeratin 19 (K19), cystic fibrosis transmembrane receptor, carbonic anhydrase II (CAII), and DBA lectin and transcriptional factors such as HNF1 $\beta$, HNF6, and SOX9. Recent work has helped to elucidate the hierarchy of transcriptional factors required to foster the formation and maturation of ductal cells during development.

$\mathrm{Gu}$ et al. showed that pancreatic ducts emerge from Pdx1expressing cells that separate from the endocrine and exocrine lineages between E9.5 and E11.5 (41). It has been demonstrated that non-islet Pdx1-positive cells display physical traits of ductal branching starting at E14.5, and there is even some evidence of these cells - which are insulin negative and K19 positive - as early as E12.5 (42). In addition, a 3D cell culture model of branching morphogenesis, using primary pancreatic duct cells, identified a transient surge of Pdx1 expression exclusive to branching cells (42). This suggests that $\mathrm{Pdx} 1$ might be involved temporally in a program of gene expression sufficient to facilitate the biochemical and morphological changes necessary for branching ductal morphogenesis, both during development and in the adult pancreas.

The Hnf6 transcription factor appears to be important in pancreatic ductal development. Mice lacking the gene ( $\mathrm{Hnf6}^{-/-}$mice) develop cysts in interlobular and intralobular pancreatic ducts but not in intercalated pancreatic ducts (43). Intriguingly, this could imply that Hnf6 function might be restricted to distinct ductal segments. Furthermore, there is an absence of primary cilia in the pancreatic tubular structures of $\mathrm{Hnf6}^{-/-}$mice (43). Conditional Hnf6 knockout in the murine pancreas recapitulates most of the features of the global knockout (44). Additionally, the pancreata from these mice display increased ductal cell proliferation and metaplasia as well as other features of pancreatitis (44).

At the time of secondary transition, the pancreatic trunk epithelium includes a progenitor population that can give rise to ductal and endocrine lineages (45). It has been demonstrated that Hnf6 is a positive regulator of the proendocrine factor ngn3 (46). Malformed ducts in global as well as conditional Hnf6 knockout mice have reduced numbers of $n g n 3^{+}$cells, along with persistent expression of endocrine markers, including glucagon, insulin, and $\operatorname{Pdx} 1$, in these ducts $(44,46)$. It is tempting to speculate that the developmental abnormalities in $\mathrm{Hnf6}^{-/-}$mice stem, in part, from the failure of ngn3-positive progenitor cells to function properly. 

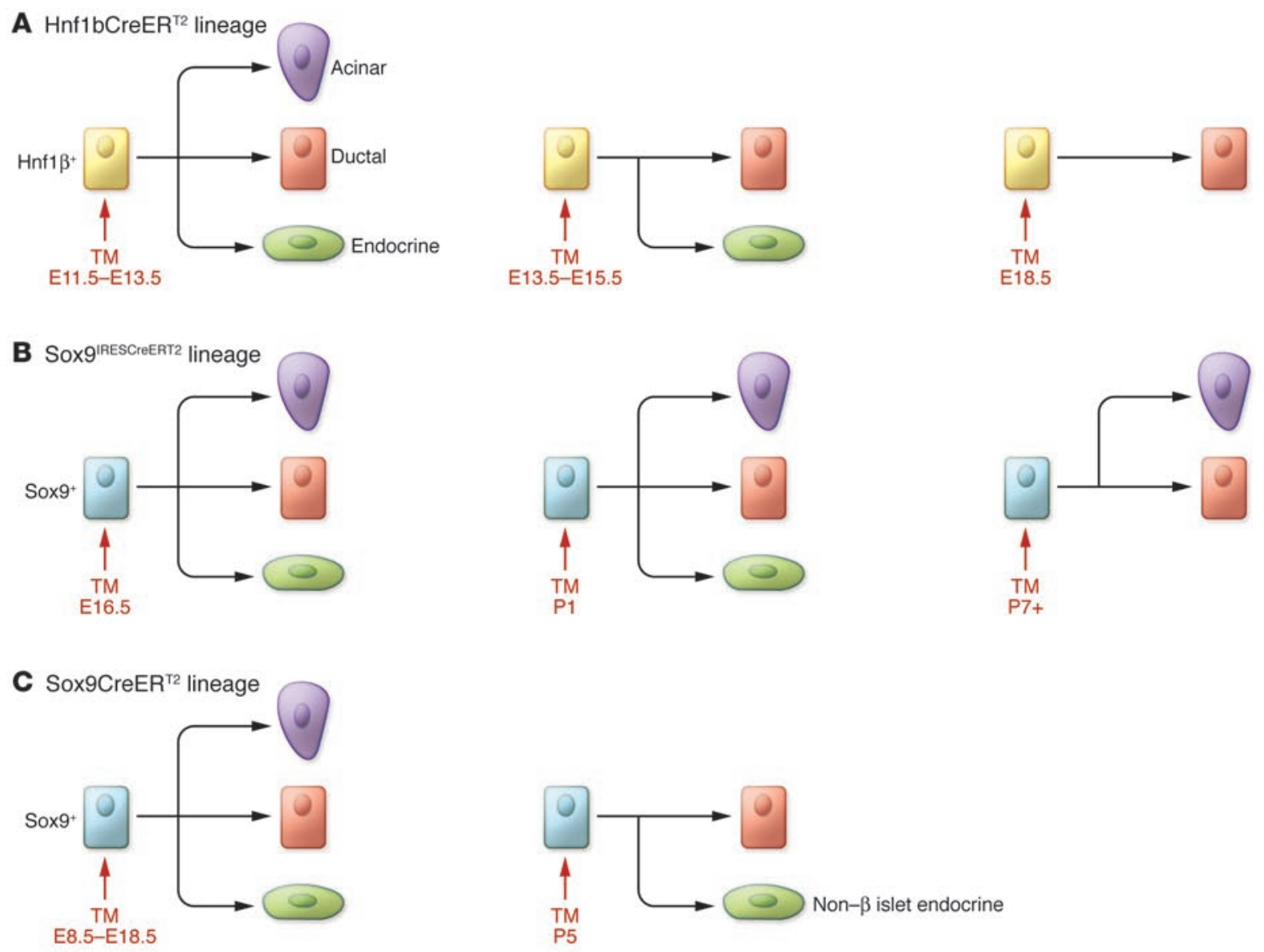

\section{Figure 2}

Role of Hnf1 $\beta$ and Sox9 in pancreatic ductal lineage specification. (A) Solar et al. (51) performed pulse-chase experiments using the Hnf1bCreER ${ }^{T 2}$;R26R mouse model. Hnf1 $\beta$-positive cells give rise to all pancreatic lineages when recombination was induced prior to secondary transition. When pulse labeled between E13.5 and E15.5, progeny cells were found only within the endocrine and ductal compartments. Induced at E18.5, Hnf1 $\beta$-positive cells are located exclusively within ducts. (B) Furuyama et al. (52) demonstrated with their Sox9/RESCreERT2;R26R model that Sox9-positive cells give rise to acinar, ductal, and endocrine cells when induced at E16.5 and P1, respectively. Any time after P7, Sox9positive cells repopulate the acinar and ductal compartment. (C) Kopp et al. (53) labeled Sox9-positive cells with a transgenic approach, using Sox9CreER ${ }^{T 2} ; R 26 R$ mice, and confirmed that Sox9-expressing cells give rise to all pancreatic lineages when induced between E8.5 and E18.5. When analyzing pancreata that were labeling at P5, recombination was observed in ducts and endocrine cells. TM, tamoxifen.

Another transcription factor critical for pancreatic ductal development and whole-organ formation is Hnf1 $\beta$ (also known as Tcf2). Hnf $1 \beta$, encoded by the maturity-onset diabetes of the young 5 (MODY5) gene, is activated downstream of Hnf6 (47), and Hnf1 $\beta$ expression is diminished in pancreatic cystic structures of $\mathrm{Hnf6}^{-/}$ mice. (43). To examine the role of Hnf1 $\beta$ in pancreas development, Haumaitre et al. generated chimeric mice by tetraploid aggregation, since $\mathrm{Hnfl}^{-/-}$mice die before gastrulation (48). These mice display agenesis of the ventral bud and rudimentary development of the dorsal pancreas, which overlaps with some features of $P t f 1 a^{-/-}$mice. In part, this may be due to direct regulation of Ptf 1 a by Hnf $1 \beta$; however, the more severe picture of perturbed pancreatic organ formation in $P t f 1 a^{-/-}$mice indicates Ptfla-independent effects of $\operatorname{Hnf} 1 \beta$ (48).

Yet another critical transcriptional factor is the Sry/HMG box transcription factor Sox9, which is essential for the maintenance of the pancreatic progenitor pool, because it stimulates proliferation and survival of progenitors. Therefore, pancreas-specific Sox9 ablation leads to hypoplasia of the gland (49). One study positions SOX9 upstream of HNF6 and HNF1 $\beta$ at the time of secondary transition (50). Interestingly, the authors show also that Sox9 directly regulates the expression of $n g n 3$, highlighting the role of Sox9 in controlling a bipotent (ductal and endocrine) progenitor population (50).

In contrast to the aforementioned gene ablation models, more recent studies have used direct cell lineage tracing to understand pancreatic lineage specification (Figure 2). Labeling Hnf1 $\beta$-expressing cells before secondary transition (E11.5-E13.5) reveals that this population gives rise to all pancreatic lineages, whereas, shortly afterward (E13.5-E15.5), Hnf1 $\beta$ cells differentiate into ductal and endocrine lineages, and labeling around E18 labels only the ductal compartment (51). More recently, Furuyama et al. (52) used a knockin lineage tracing approach to show that even after secondary transition (E16.5 or as late as P1), Sox9-positive cells contribute to the ductal, acinar, and endocrine lineages. After induction at P7, labeled cells were found exclusively within the ductal and acinar compartments. This holds true in their model system even in older mice (eight weeks), suggesting Sox9-positive ductal cells or centroacinar cells maintain the exocrine compartment (52). 
Sander and colleagues obtained different results with a Sox9CreER $R^{T 2}$ transgenic mouse model. Indeed, their pulse-chase experiments confirmed that inducing at any time between E8.5 and E18.5 labels all pancreatic lineages (53). By contrast, at P5, Sox9-positive cells give rise to ductal cells and to few endocrine cells. However, although a small percentage of insulin-positive cells are lineage labeled, these fail to give rise to new $\beta$ cells in the time course of the experiment. Therefore, Sox9-positive cells do not contribute to the $\beta$ cell compartment during this early postnatal period in these experiments (53). Overall, these discordant results are likely due to differences between the two mouse models and leave some room for differing interpretations of the role of Sox9 in the specification of pancreatic lineages.

It seems to some extent that the HNF1 $\beta$-positive population differs from the Sox9-positive population. Kopp et al. demonstrated that some of the Sox9-positive cells at E14.5 are HNF1 $\beta$ negative; however, they coexpress Ptf1a, Nkx6.1, and Pdx1 and are localized at the interface between the trunk and tip, a region that might represent a niche for progenitor cells (53). Of note, evidence from in vitro experiments shows that Sox9 interacts with Hnf1 $\beta$, Hnf6, and Foxa2, and these interactions could modulate the action of each transcription factor in pancreatic development. In turn, both Foxa2 and Hnf1b regulate Sox 9 expression, demonstrating feedback loops between these genes $(49,50)$.

\section{Plasticity of ductal cells: a source of islet cells?}

Of intense interest in the study of ductal cells has been their potential capacity to give rise to islet cells. If possible, this would be another vehicle to generate islet cells for transplantation as well as a potential treatment of diabetes mellitus. Insights into ductal cell plasticity have been gained through in vitro and in vivo approaches. For example, ligation of the pancreatic duct in the adult rat fosters duct- to islet-cell differentiation (54). Human ductal tissue in vitro can be expanded to insulin-positive cells (55). However, genetically based in vivo studies have yielded divergent results. One study concluded that $\beta$ cell progenitors can be activated in the injured adult mouse pancreas and are located in the ductal lining (56). Differentiation of these adult endocrine pancreatic progenitor cells was ngn3 dependent, and all islet cell types emerged from this population. The authors demonstrated $\beta$ cell proliferation both in situ and in embryonic explant culture systems (56). Another study used the pancreatic ductal-specific human carbonic anhydrase II (CAII) promoter with an inducible Cre/lox model in lineage tracing studies. CAII-expressing cells within the pancreas appear to act as progenitors that give rise to both new islets and acini, normally after birth and after injury (pancreatic duct ligation [PDL]) (57).

These findings, however, have been challenged. For example, Hnf1 $\beta$-positive cells do not give rise to acinar or endocrine cells during the neonatal period, during a 6-month period, or during induction of different types of injury (51). In another study, a subset of Sox9-positive cells start to express ngn3 after PDL; however, this population fails to contribute to the emergence of the endocrine lineage (53). One possible conclusion that emerges from these divergent studies is that a subpopulation of ductal cells or cells lining the ductal epithelium, marked by endocrine marker(s), is able to give rise to islet cells in the adult, but this activation requires some form of injury, and such a population is not genetically labeled by either Hnf $1 \beta$ or Sox9. Additionally, preexisting $\beta$ cells are the major source of new $\beta$ cells during adult life and after partial pancreatectomy in mice (54-58), supporting the premise that $\beta$ cells arise from self-duplication (58-62). These results sug- gest that major hurdles remain before the potential plasticity of ductal cells can be exploited as a source of new $\beta$ cells.

\section{ADM: insights into ductal cell reprogramming and neoplastic transformation}

Metaplasia is defined by the conversion or replacement of one differentiated cell type with another in the context of a given tissue. In some tissues, metaplasia is associated with an increased risk of cancer. Investigation of metaplasia might also provide clues to the cell of origin of cancer. To that end, pancreatic acinar cells have the capacity to undergo metaplasia to a ductal cell phenotype in the setting of acute or chronic inflammation, representing an important link to pancreatic ductal adenocarcinoma (PDAC). ADM might represent reprogramming of a progenitor population, direct transdifferentiation of acinar cells to ductal cells, or transdifferentiation via an intermediate cell type (potentially a progenitor cell). Lineage tracing suggests ADM occurs in vivo but occurs only in a subset of metaplastic lesions (63). Exocrine epithelial explants lose acinar cells in culture with expansion of ductal cells (64-67); however, these results could be due to potential survival advantages of ductal cells in culture.

The molecular basis underlying the development of ADM involves a number of seemingly diverse pathways and molecules. One common theme in divergent studies of ADM is the importance of TGF- $\alpha$ and EGFR signaling. Transgenic mice with Tgfa overexpression in the exocrine pancreas progress to a phenotype marked by duct-like cells and tubular structures (64). The importance of EGFR signaling in ADM has been underscored in other studies as well (68-70). Acinar-enriched epithelial explants were isolated from a mouse model designed for acinar-specific lineage tracing, involving Cre/lox-mediated $\beta$-gal expression, and cultured subsequently in the presence of recombinant human TGF- $\alpha$ (71). At the time of isolation, $\beta$-gal staining was observed exclusively in acinar cells, and no $\beta$-gal activity was observed in cells expressing duct-specific keratins. After culturing epithelial explants for five days in the presence of TGF- $\alpha$, cystic ductal epithelial structures formed that were positive for $\beta$-gal activity (71). Houbracken and colleagues very recently showed that human acinar cells display an equal amount of plasticity through lineage tracing (72). Interestingly, after one week of culture, almost all of the surviving acinar cells expressed ductal markers. This could be prevented partially by inhibiting MAPK signaling (72).

TGF- $\alpha$-mediated engagement of EGFR results in the activation of downstream signaling cascades that might involve Kras activation. Preneoplastic and neoplastic lesions in the pancreas harbor mutations in the Kras oncogene, resulting in constitutive activation of downstream signaling cascades $(73,74)$. Such mutations are critical in the induction of neoplastic lesions. Acinar-specific expression of activated Kras is one of several mouse models of PDAC. Analysis of such mice (LSL-Kras ${ }^{G 12 D /+} / \mathrm{Ptf1aCre} /+$ mice) at four weeks of age reveals that the predominant cellular alteration in the exocrine pancreas is acinar metaplasia, in which individual acini consist of acinar cells and duct-like cells (75). Metaplastic acinar structures are highly proliferative, express Notch target genes, and exhibit mosaic expression patterns for EGFR, ErbB2, and pErk, reminiscent of the PDAC precursors, pancreatic intraepithelial neoplasia (PanIN) lesions $(71,75,76)$. Kras activation in mature acinar cells induces PanIN lesions identical to those observed upon ubiquitous Kras activation, and Notch signaling promotes both initiation and progression of these acinar-derived PanINs (77). At the cellular level, Notch/Kras coactivation promotes rapid reprogramming of acinar cells to a duct-like phenotype. 


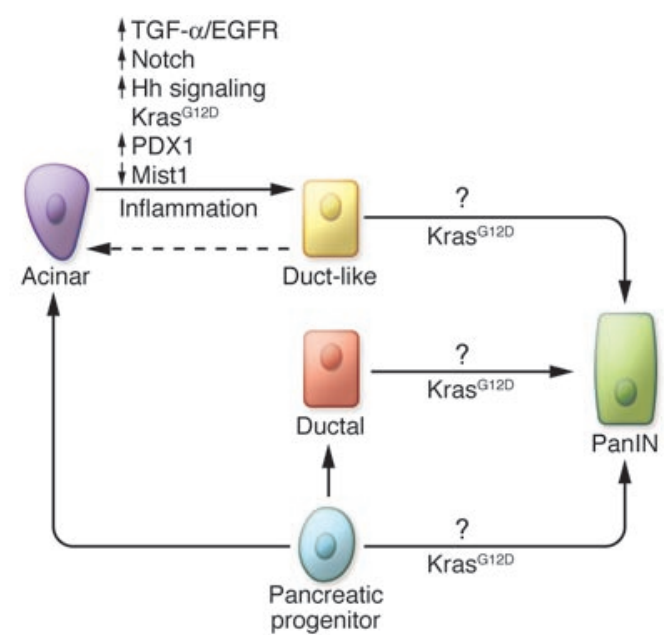

Figure 3

ADM and PanIN — roles of potential pathways. In the adult mouse pancreas, ADM may be triggered by inflammation and other factors. Known associations with ADM include, but are not limited to, extracellular cues - TGF- $\alpha$, Hedgehog signaling, Notch signaling - and intracellular alterations mediated by $\mathrm{Kras}^{\mathrm{G} 12 \mathrm{D}}$ activation, including induction of $\mathrm{Pdx1}$, induction of TNF- $\alpha$, and decreased Mist1 expression. Ductal cells or duct-like cells are replaced by healthy acinar cells during recovery from injury. ADM might represent a precursor lesion to PanIN and constitute a cell of origin for PanIN. The cell of origin for PanIN might be a ductal cell or a distinct yet-to-be-identified progenitor cell.

Another theme in ADM involves changes in the transcriptional network. Spontaneous ADM has been described in vitro, accompanied by the induction of Pdx1 expression during culture of acinar cells (66). Based upon the finding that transgenic mice with $\mathrm{Tg} f a$ overexpression develop ADM and an expansion of Pdx1-positive epithelial cells (70), a more direct role for Pdx1 in ADM has been suggested. Indeed, transgenic expression of Pdx1 in the Ptf1a domain (35) results in the replacement of acinar cells by duct-like structures, and, furthermore, this requires Stat 3 activation (78).

Another relevant transcription factor is Mist1. Mist1 functions as a homodimer, and its loss results in ADM in vitro, with accompanying induction of K19 and K20 (79). Transgenic mice expressing a dominant-negative Mist 1 undergo ADM in vivo (79). Collectively, these studies suggest that loss of Mist 1 initiates metaplasia and that Pdx 1 expression fosters ADM, perhaps mediated by Stat 3 as a potential target of $\mathrm{Pdx} 1$.

One unifying (perhaps simplified) model underlying ADM involves TGF- $\alpha$ engagement of EGFR, subsequent EGFR-mediated activation of downstream signaling effectors (e.g., Kras, MAPK, Pten) (80), and, ultimately, alteration (induction or suppression) of key transcription factors, such as Mist1, Pdx1, and Stat3. In addition to EGFR signaling, Notch signaling (manifested by Hes1 expression) suppresses the acinar cell phenotype (16) and favors ADM (75). It is also known that Notch is required for TGF- $\alpha$-mediated effects in the pancreas (76). Thus, the acinar cell state is Mist 1 positive, Pdx1 negative, and Hes 1 negative, and, with the transition to the ductal state, metaplastic cells are increasingly Mist 1 negative, $\mathrm{Pdx} 1$ positive, and Hes 1 positive. This model would presuppose that ADM is critical in neoplastic transformation, abetted by a proinflammatory state $(81,82)$.

\section{Ductal cells and their relationship to PanIN and PDAC}

The most common subtype of human pancreatic malignancy is PDAC. It has been established that PDAC is preceded by the evolution of precursor lesions, PanIN 1A/B, 2, and $3(83,84)$, and, under certain conditions, ADM might be critical for the development of PanIN lesions $(70,71)$. PanIN lesions can occur in the setting of chronic pancreatitis and might also be age dependent in the background of otherwise normal pancreatic architecture at the time of autopsy. An unresolved question relates to the cell of origin for PanIN and PDAC. Genetically engineered mouse models using mutant Kras support the notion that ADM might represent a prerequisite for PanIN and PDAC development $(70,71)$. In addition, the chronic administration of cerulein to mutant Kras mice accelerates PDAC lesions, suggesting that inflammation is important in mediating tumorigenesis (80), which is influenced by different cell types in the tumor microenvironment (85).

Conceivably, ADM yields a cell type with ductal features that is susceptible to the effects of Kras activation and other genetic alterations (Figure 3). Intriguingly, spontaneous conversion of acinar cells to PanIN lesions in the mouse can occur by direct targeting of acinar cells with $\mathrm{Kras}^{\mathrm{G} 12 \mathrm{D}}$ in the absence of injury or inflammation (86). However, the development of PanIN lesions in vivo may involve the emergence of a progenitor population that is either an indirect or direct precursor to cells that will contribute to a PanIN (Figure 3). This progenitor population would necessarily express $\mathrm{Pdx} 1$, which is normally low or absent in ductal cells. It is possible that a resident progenitor population exists among ductal cells or centroacinar cells, which undergoes neoplastic transformation without ADM. The existence of such a population is suggested by the finding that a subset of adult mouse centroacinar cells/terminal duct cells harbor high aldehyde dehydrogenase isoform 1 (ALDH1) enzymatic activity, which is important in retinoic acid metabolism and has been associated with stem and progenitor cells in a variety of tissue types (87). Isolation of Aldh1-positive cells by flow cytometry reveals enrichment of Sca1, Sdf1, c-Met, Nestin, and Sox 9 (87). These cells form "pancreatospheres" in culture, with a capacity for endocrine and exocrine differentiation (suggesting plasticity), and independently expand in the face of chronic epithelial injury. As a related but separate consideration, whether such a population may participate in the eventual development of pancreatic cancer stem cells, marked by CD133, CXCR4, CD44, CD24, and epithelial-specific antigen (ESA) expression $(88,89)$, is not clear. While it is believed that non-islet cells are the cells of origin for PanIN and PDAC lesions, it has been suggested that chronic pancreatic injury can alter the endocrine fate of $\beta$ cells, which are usually resistant to the effects of oncogenic insults, and allow them to serve as the cells of origin for exocrine neoplasia (90).

Definitive proof for a ductal cell or centroacinar cell of origin for PanIN and PDAC will require the generation and characterization of appropriate cell-type specific promoters with endogenous Kras activation (with lineage tracing strategies), possibly coupled to other genetic alterations (e.g., mutant p53). These approaches could then be placed into the context of existing genetically engineered mouse models of PDAC that use Pdx1-cre or Ptf1a-cre.

\section{Summary and future directions}

The ductal tree evolves during mouse embryogenesis from a common progenitor pool that is fated to evolve into the endocrine and exocrine lineages. First evident at E14-E15, the ductal structures mature by E17-E18 (42). Pdx1, EphB signaling, and Cdc42 are important in pancreatic ductal branching morphogenesis (42, 
91, 92). Elegant genetically engineered mouse models capitalizing upon the expression patterns of $\operatorname{Hnf} 1 \beta, \mathrm{Hnf} 6$, and Sox 9 have yielded somewhat divergent results for the capacity of ductal cells to give rise to other lineages during development in the postnatal period and in young mice. In the adult mouse pancreas, it is clear that ductal cells, like islet and acinar cells, are relatively quiescent under homeostatic conditions. However, injurious conditions may reveal a restricted or transient capacity of acinar cells to evolve into ductal cells or ADM. ADM is an important precursor to PanIN lesions, and PanIN lesions progress to PDAC, at least in the mouse. Whether ADM results from direct metaplasia or perhaps transdifferentiation, or whether either process occurs through an intermediate progenitor cell, remains unresolved. It should be noted that the development of PanIN lesions may not require ADM. The adult pancreas may well harbor a low-frequency progenitor population, perhaps resident among centroacinar cells or among ductal cells, a population that may be unmasked during injury and inflammation and may even have the capacity to give rise to islet cells.

Questions remain about the transcriptional regulation of gene expression in ductal cells, which might have shared mechanisms with genes expressed in acinar and islet cells (93-95), and the differences between terminal ducts and the main duct as well as about whether ductal cells can serve as the cells of origin for PanIN/ PDAC and whether progenitor cells might reside in the ductal tree itself. Furthermore, in recent years, insights have been gained into the clinical, histopathological, and genetic characteristics of mucinous cystic lesions in the pancreas (intraductal papillary mucinous neoplasm [IPMN], mucinous cystic neoplasm), which carry a low malignant potential and might represent developmental derangements in the ductal tree, although they manifest in late adulthood (96-101). For example, comparisons between IPMN and PDAC might help to elucidate whether the location of the lesion within the ductal system contributes to the pathogenesis of the type of malignancy. These studies underscore the necessity for an increased understanding of ductal cell biology to inform our research in fields as diverse as development and tumorigenesis.

\section{Acknowledgments}

This work was supported by NIH grant R01 DK060694 (to M. Reichert and A.K. Rustgi), National Pancreas Foundation (to M. Reichert), NIH Center for Molecular Studies in Digestive and Liver Diseases (P30-DK050306), and NIH Diabetes and Endocrinology Research Center (P30-DK019525).

Address correspondence to: Anil K. Rustgi, GI Division, 600 CRB, 415 Curie Blvd., University of Pennsylvania School of Medicine, Philadelphia, Pennsylvania 19104, USA. Phone: 215.898.0154; Fax: 215.573.5412; E-mail: anil2@mail.med.upenn.edu.
1. Cano DA, Hebrok M, Zenker M. Pancreatic development and disease. Gastroenterology. 2007; 132(2):745-762.

2. Means AL, Leach SD. Lineage commitment and cellular differentiation in exocrine pancreas. Pancreatology. 2001;1(6):587-596.

3. Bonal C, Herrera PL. Genes controlling pancreas ontogeny. Int J Dev Biol. 2008;52(7):823-835.

4. Gittes GK. Developmental biology of the pancreas: a comprehensive review. Dev Biol. 2009;326(1):4-35.

5. Kim SK, MacDonald RJ. Signaling and transcriptional control of pancreatic organogenesis. Curr Opin Genet Dev. 2002;12(5):540-547.

6. Pan FC, Wright C. Pancreas organogenesis: from bud to plexus to gland. Dev Dyn. 2011;240(3):530-565.

7. MacDonald RJ, Swift GH, Real FX. Transcriptional control of acinar development and homeostasis. Prog Mol Biol Transl Sci. 2010;97:1-40.

8. Puri S, Hebrok M. Cellular plasticity within the pancreas--lessons learned from development. Dev Cell. 2010;18(3):342-356.

9. Wandzioch E, Zaret KS. Dynamic signaling network for the specification of embryonic pancreas and liver progenitors. Science. 2009;324(5935):1707-1710.

10. Pictet RL, Clark WR, Williams RH, Rutter WJ. An ultrastructural analysis of the developing embryonic pancreas. Dev Biol. 1972;29(4):436-467.

11. Hebrok M, Kim SK, Melton DA. Notochord repression of endodermal Sonic hedgehog permits pancreas development. Genes Dev. 1998;12(11):1705-1713.

12. Hebrok M, Kim SK, St Jacques B, McMahon AP, Melton DA. Regulation of pancreas development by hedgehog signaling. Development. 2000; 127(22):4905-4913.

13. Lau J, Hebrok M. Hedgehog signaling in pancreas epithelium regulates embryonic organ formation and adult beta-cell function. Diabetes. 2010; 59(5):1211-1221.

14. Apelqvist A, et al. Notch signalling controls pancreatic cell differentiation. Nature. 1999; 400(6747):877-881.

15. Esni F, Johansson BR, Radice GL, Semb H. Dorsal pancreas agenesis in N-cadherin- deficient mice. Dev Biol. 2001;238(1):202-212.

16. Hald J, Hjorth JP, German MS, Madsen OD, Serup $P$, Jensen J. Activated Notch1 prevents differentia- tion of pancreatic acinar cells and attenuate endocrine development. Dev Biol. 2003;260(2):426-437.

17. Murtaugh LC, Stanger BZ, Kwan KM, Melton DA. Notch signaling controls multiple steps of pancreatic differentiation. Proc Natl Acad Sci U S A. 2003; 100(25):14920-14925.

18. Burlison JS, Long Q, Fujitani Y, Wright CV, Magnuson MA. Pdx-1 and Ptf1a concurrently determine fate specification of pancreatic multipotent progenitor cells. Dev Biol. 2008;316(1):74-86.

19. Gannon M, Herrera PL, Wright CV. Mosaic Cremediated recombination in pancreas using the $\mathrm{pdx}$ 1 enhancer/promoter. Genesis. 2000;26(2):143-144.

20. Jonsson J, Carlsson L, Edlund T, Edlund H. Insulinpromoter-factor 1 is required for pancreas development in mice. Nature. 1994;371(6498):606-609.

21. Fujitani Y, et al. Targeted deletion of a cis-regulatory region reveals differential gene dosage requirements for $\mathrm{Pdx} 1$ in foregut organ differentiation and pancreas formation. Genes Dev. 2006; 20(2):253-266.

22. Gannon $M$, et al. pdx-1 function is specifically required in embryonic beta cells to generate appropriate numbers of endocrine cell types and maintain glucose homeostasis. Dev Biol. 2008; 314(2):406-417.

23. Gannon M, Gamer LW, Wright CV. Regulatory regions driving developmental and tissue-specific expression of the essential pancreatic gene $\mathrm{pdx} 1$. Dev Biol. 2001;238(1):185-201.

24. Murtaugh LC, Melton DA. Genes, signals, and lineages in pancreas development. Annu Rev Cell Dev Biol. 2003;19:71-89.

25. Offield MF, et al. PDX-1 is required for pancreatic outgrowth and differentiation of the rostral duodenum. Development. 1996;122(3):983-995.

26. Schonhoff SE, Giel-Moloney M, Leiter AB. Neurogenin 3-expressing progenitor cells in the gastrointestinal tract differentiate into both endocrine and nonendocrine cell types. Dev Biol. 2004;270(2):443-454.

27. Apelqvist A, Ahlgren U, Edlund H. Sonic hedgehog directs specialised mesoderm differentiation in the intestine and pancreas. Curr Biol. 1997;7(10):801-804.

28. Murtaugh LC. The what, where, when and how of Wnt/beta-catenin signaling in pancreas development. Organogenesis. 2008;4(2):81-86.
29. Li Z, et al. Multifaceted pancreatic mesenchymal control of epithelial lineage selection. Dev Biol. 2004; 269(1):252-263.

30. Miralles F, Czernichow P, Scharfmann R. Follistatin regulates the relative proportions of endocrine versus exocrine tissue during pancreatic development. Development. 1998;125(6):1017-1024.

31. Bhushan A, et al. Fgf10 is essential for maintaining the proliferative capacity of epithelial progenitor cells during early pancreatic organogenesis. Development. 2001;128(24):5109-5117.

32. Cras-Meneur C, Elghazi L, Czernichow P, Scharfmann R. Epidermal growth factor increases undifferentiated pancreatic embryonic cells in vitro: a balance between proliferation and differentiation. Diabetes. 2001;50(7):1571-1579.

33. Hart AW, Baeza N, Apelqvist A, Edlund H. Attenuation of FGF signalling in mouse beta-cells leads to diabetes. Nature. 2000;408(6814):864-868

34. Miralles F, Battelino T, Czernichow P, Scharfmann $\mathrm{R}$. TGF-beta plays a key role in morphogenesis of the pancreatic islets of Langerhans by controlling the activity of the matrix metalloproteinase MMP2. J Cell Biol. 1998;143(3):827-836.

35. Kawaguchi Y, Cooper B, Gannon M, Ray M, MacDonald RJ, Wright CV. The role of the transcriptional regulator Ptf1a in converting intestinal to pancreatic progenitors. Nat Genet. 2002;32(1):128-134.

36. Lin JW, et al. Differential requirement for ptf1a in endocrine and exocrine lineages of developing zebrafish pancreas. Dev Biol. 2004;270(2):474-486.

37. Ornitz DM, Palmiter RD, Hammer RE, Brinster RL, Swift GH, MacDonald RJ. Specific expression of an elastase-human growth hormone fusion gene in pancreatic acinar cells of transgenic mice. Nature. 1985;313(6003):600-602.

38. Krapp A, et al. The bHLH protein PTF1-p48 is essential for the formation of the exocrine and the correct spatial organization of the endocrine pancreas. Genes Dev. 1998;12(23):3752-3763.

39. Masui T, Long Q, Beres TM, Magnuson MA, MacDonald RJ. Early pancreatic development requires the vertebrate Suppressor of Hairless (RBPJ) in the PTF1 bHLH complex. Genes Dev. 2007;21(20):2629-2643.

40. Pin CL, Rukstalis JM, Johnson C, Konieczny SF. The bHLH transcription factor Mist 1 is required to 
maintain exocrine pancreas cell organization and acinar cell identity. J Cell Biol. 2001;155(4):519-530.

41. Gu G, Dubauskaite J, Melton DA. Direct evidence for the pancreatic lineage: NGN3+ cells are islet progenitors and are distinct from duct progenitors. Development. 2002;129(10):2447-2457.

42. Wescott MP, et al. Pancreatic ductal morphogenesis and the Pdx1 homeodomain transcription factor. Mol Biol Cell. 2009;20(22):4838-4844.

43. Pierreux CE, et al. The transcription factor hepatocyte nuclear factor- 6 controls the development of pancreatic ducts in the mouse. Gastroenterology. 2006;130(2):532-541.

44. Zhang H, et al. Multiple, temporal-specific roles for HNF6 in pancreatic endocrine and ductal differentiation. Mech Dev. 2009;126(11-12):958-973.

45. Zhou Q, Law AC, Rajagopal J, Anderson WJ, Gray PA, Melton DA. A multipotent progenitor domain guides pancreatic organogenesis. Dev Cell. 2007; 13(1):103-114

46. Jacquemin $P$, et al. Transcription factor hepatocyte nuclear factor 6 regulates pancreatic endocrine cell differentiation and controls expression of the proendocrine gene ngn3. Mol Cell Biol. 2000; 20(12):4445-4454.

47. Maestro MA, et al. Hnf6 and Tcf2 (MODY5) are linked in a gene network operating in a precursor cell domain of the embryonic pancreas. Hum Mol Genet. 2003;12(24):3307-3314.

48. Haumaitre C, Barbacci E, Jenny M, Ott MO, Gradwohl G, Cereghini S. Lack of TCF2/vHNF1 in mice leads to pancreas agenesis. Proc Natl Acad Sci US A. 2005;102(5):1490-1495.

49. Seymour PA, et al. SOX9 is required for maintenance of the pancreatic progenitor cell pool. Proc Natl Acad Sci U S A. 2007;104(6):1865-1870.

50. Lynn FC, Smith SB, Wilson ME, Yang KY, Nekrep $\mathrm{N}$, German MS. Sox 9 coordinates a transcriptional network in pancreatic progenitor cells. Proc Natl Acad Sci U S A. 2007;104(25):10500-10505.

51. Solar M, et al. Pancreatic exocrine duct cells give rise to insulin-producing beta cells during embryogenesis but not after birth. Dev Cell. 2009;17(6):849-860.

52. Furuyama $\mathrm{K}$, et al. Continuous cell supply from a Sox9-expressing progenitor zone in adult liver, exocrine pancreas and intestine. Nat Genet. 2011 43(1):34-41.

53. Kopp JL, et al. Sox9+ ductal cells are multipotent progenitors throughout development but do not produce new endocrine cells in the normal or injured adult pancreas. Development. 2011;138(4):653-665.

54. Wang RN, Kloppel G, Bouwens L. Duct- to isletcell differentiation and islet growth in the pancreas of duct-ligated adult rats. Diabetologia. 1995 38(12):1405-1411.

55 . Bonner-Weir $\mathrm{S}$, et al. In vitro cultivation of human islets from expanded ductal tissue. Proc Natl Acad Sci U S A. 2000;97(14):7999-8004.

56. Xu X, et al. Beta cells can be generated from endogenous progenitors in injured adult mouse pancreas. Cell. 2008;132(2):197-207.

57. Inada A, et al. Carbonic anhydrase II-positive pancreatic cells are progenitors for both endocrine and exocrine pancreas after birth. Proc Natl Acad Sci U S A. 2008;105(50):19915-19919.

58. Brennand K, Huangfu D, Melton D. All beta cells contribute equally to islet growth and maintenance. PLoS Biol. 2007;5(7):e163.

59. Dor Y, Brown J, Martinez OI, Melton DA. Adult pancreatic beta-cells are formed by self-duplication rather than stem-cell differentiation. Nature. 2004;429(6987):41-46

60. Lee CS, De Leon DD, Kaestner KH, Stoffers DA. Regeneration of pancreatic islets after partial pancreatectomy in mice does not involve the reactivation of neurogenin-3. Diabetes. 2006;55(2):269-272.

61. Nir T, Melton DA, Dor Y. Recovery from diabetes in mice by beta cell regeneration. J Clin Invest.
2007;117(9):2553-2561.

62. Teta M, Rankin MM, Long SY, Stein GM, Kushner JA. Growth and regeneration of adult beta cells does not involve specialized progenitors. Dev Cell. 2007;12(5):817-826.

63. Strobel $O$, et al. In vivo lineage tracing defines the role of acinar-to-ductal transdifferentiation in inflammatory ductal metaplasia. Gastroenterology. 2007;133(6):1999-2009.

64. De Lisle RC, Logsdon CD. Pancreatic acinar cells in culture: expression of acinar and ductal antigens in a growth-related manner. Eur J Cell Biol. 1990;51(1):64-75.

65. Githens S, Schexnayder JA, Moses RL, Denning GM, Smith JJ, Frazier ML. Mouse pancreatic acinar/ductular tissue gives rise to epithelial cultures that are morphologically, biochemically, and functionally indistinguishable from interlobular duct cell cultures. In Vitro Cell Dev Biol Anim. 1994; 30A(9):622-635.

66. Rooman I, Heremans Y, Heimberg H, Bouwens L. Modulation of rat pancreatic acinoductal transdifferentiation and expression of PDX-1 in vitro. Diabetologia. 2000;43(7):907-914.

67. Sphyris N, Logsdon CD, Harrison DJ. Improved retention of zymogen granules in cultured murine pancreatic acinar cells and induction of acinarductal transdifferentiation in vitro. Pancreas. 2005; 30(2):148-157.

68. Means AL, et al. Overexpression of heparin-binding EGF-like growth factor in mouse pancreas results in fibrosis and epithelial metaplasia. Gastroenterology. 2003;124(4):1020-1036.

69. Sandgren EP, Luetteke NC, Palmiter RD, Brinster RL, Lee DC. Overexpression of TGF alpha in transgenic mice: induction of epithelial hyperplasia, pancreatic metaplasia, and carcinoma of the breast. Cell. 1990;61(6):1121-1135.

70. Song SY, et al. Expansion of Pdx1-expressing pancreatic epithelium and islet neogenesis in transgenic mice overexpressing transforming growth factor alpha. Gastroenterology. 1999;117(6):1416-1426.

71. Means AL, et al. Pancreatic epithelial plasticity mediated by acinar cell transdifferentiation and generation of nestin-positive intermediates. Development. 2005;132(16):3767-3776.

72. Houbracken I, et al. Lineage tracing evidence for transdifferentiation of acinar to duct cells and plasticity of human pancreas. Gastroenterology. 2011; 141(2):731-741.

73. Aguirre AJ, et al. Activated Kras and Ink4a/Arf deficiency cooperate to produce metastatic pancreatic ductal adenocarcinoma. Genes Dev. 2003; 17(24):3112-3126.

74. Hingorani SR, et al. Preinvasive and invasive ductal pancreatic cancer and its early detection in the mouse. Cancer Cell. 2003;4(6):437-450.

75. Zhu L, Shi G, Schmidt CM, Hruban RH, Konieczny SF. Acinar cells contribute to the molecular heterogeneity of pancreatic intraepithelial neoplasia. AmJ Pathol. 2007;171(1):263-273.

76. Miyamoto Y, et al. Notch mediates TGF alphainduced changes in epithelial differentiation during pancreatic tumorigenesis. Cancer Cell. 2003; 3(6):565-576

77. De La OJ, et al. Notch and Kras reprogram pancreatic acinar cells to ductal intraepithelial neoplasia. Proc Natl Acad Sci U S A. 2008;105(48):18907-18912.

78. Miyatsuka T, et al. Persistent expression of PDX1 in the pancreas causes acinar-to-ductal metaplasia through Stat3 activation. Genes Dev. 2006; 20(11):1435-1440

79. Zhu L, Tran T, Rukstalis JM, Sun P, Damsz B, Konieczny SF. Inhibition of Mist1 homodimer formation induces pancreatic acinar-to-ductal metaplasia. Mol Cell Biol. 2004;24(7):2673-2681.

80. Stanger BZ, et al. Pten constrains centroacinar cell expansion and malignant transformation in the pancreas. Cancer Cell. 2005;8(3):185-195.

81. Lesina M, et al. Stat3/Socs3 activation by IL-6 transsignaling promotes progression of pancreatic intraepithelial neoplasia and development of pancreatic cancer. Cancer Cell. 2011;19(4):456-469.

82. Fukuda A, et al. Stat 3 and MMP7 contribute to pancreatic ductal adenocarcinoma initiation and progression. Cancer Cell. 2011;19(4):441-455.

83. Hruban RH, et al. Pathology of genetically engineered mouse models of pancreatic exocrine cancer: consensus report and recommendations. Cancer Res. 2006;66(1):95-106.

84. Hruban RH, et al. Pancreatic intraepithelial neoplasia: a new nomenclature and classification system for pancreatic duct lesions. Am J Surg Pathol. 2001; 25(5):579-586

85. Clark CE, Hingorani SR, Mick R, Combs C, Tuveson DA, Vonderheide RH. Dynamics of the immune reaction to pancreatic cancer from inception to invasion. Cancer Res. 2007;67(19):9518-9527.

86 . Habbe N, et al. Spontaneous induction of murine pancreatic intraepithelial neoplasia (mPanIN) by acinar cell targeting of oncogenic Kras in adult mice. Proc Natl Acad Sci US A. 2008;105(48):18913-18918.

87. Rovira M, Scott SG, Liss AS, Jensen J, Thayer SP, Leach SD. Isolation and characterization of centroacinar/terminal ductal progenitor cells in adult mouse pancreas. Proc Natl Acad Sci U S A. 2010; 107(1):75-80

88. Hermann PC, et al. Distinct populations of cancer stem cells determine tumor growth and metastatic activity in human pancreatic cancer. Cell Stem Cell. 2007;1(3):313-323.

89. Li C, et al. Identification of pancreatic cancer stem cells. Cancer Res. 2007;67(3):1030-1037.

90. Gidekel Friedlander SY, et al. Context-dependent transformation of adult pancreatic cells by oncogenic K-Ras. Cancer Cell. 2009;16(5):379-389.

91. Kesavan G, et al. Cdc42-mediated tubulogenesis controls cell specification. Cell. 2009;139(4):791-801.

92. Villasenor A, Chong DC, Henkemeyer M, Cleaver O. Epithelial dynamics of pancreatic branching morphogenesis. Development. 2010;137(24):4295-4305.

93. Deramaudt TB, Sachdeva MM, Wescott MP, Chen Y, Stoffers DA, Rustgi AK. The PDX1 homeodomain transcription factor negatively regulates the pancreatic ductal cell-specific keratin 19 promoter. J Biol Chem. 2006;281(50):38385-38395.

94. Schreiber FS, et al. Successful growth and characterization of mouse pancreatic ductal cells: functional properties of the Ki-RAS(G12V) oncogene. Gastroenterology. 2004;127(1):250-260.

95. von Burstin J, Reichert M, Wescott MP, Rustgi AK. The pancreatic and duodenal homeobox protein PDX-1 regulates the ductal specific keratin 19 through the degradation of MEIS1 and DNA binding. PLoS One. 2010;5(8):e12311.

96. Bardeesy N, et al. Smad4 is dispensable for normal pancreas development yet critical in progression and tumor biology of pancreas cancer. Genes Dev. 2006;20(22):3130-3146

97. Fernandez-del Castillo C, Adsay NV. Intraductal papillary mucinous neoplasms of the pancreas. Gastroenterology. 2010;139(3):708-713.

98. Hingorani SR. Location, location, location: precursors and prognoses for pancreatic cancer. Gastroenterology. 2007;133(1):345-350.

99. Izeradjene K, et al. Kras(G12D) and Smad4/Dpc4 haploinsufficiency cooperate to induce mucinous cystic neoplasms and invasive adenocarcinoma of the pancreas. Cancer Cell. 2007;11(3):229-243.

100. Rodriguez JR, et al. Branch-duct intraductal papillary mucinous neoplasms: observations in 145 patients who underwent resection. Gastroenterology. 2007;133(1):72-79.

101. Wilentz RE, Albores-Saavedra J, Hruban RH. Mucinous cystic neoplasms of the pancreas. Semin Diagn Pathol. 2000;17(1):31-42. 